\title{
LA CIENCIA DE GÉNERO SEGÚN ANGÉLICA GORODISCHER
}

\author{
POR \\ Nicole L. Sparling \\ Central Michigan University
}

Me interesa su opinión sobre ese fenómeno de. No sé cómo

llamarlo, y eso me molesta.

Angélica Gorodischer, "Los embriones del violeta"

La incapacidad del personaje, el cronista Leo Sessler, para encontrar una palabra adecuada para describir el mecanismo y la naturaleza de la reproducción en el planeta Salari II que tiene su origen misterioso en las manchas violeta, refleja no sólo el efecto de extrañamiento cognitivo en el lenguaje mismo, sino que también representa la función del género (genre) de ciencia ficción y del género (gender) como categoría analítica en la obra de ciencia ficción más antologizada y celebrada de Angélica Gorodischer. En primer lugar, mi investigación pregunta qué significa que Gorodischer se proclame feminista y que desdibuje las fronteras entre ciencia ficción dura y ciencia ficción blanda en "Los embriones del violeta" de su colección de cuentos Bajo las jubeas en flor (1973). En segundo lugar, sostengo que un análisis del concepto titular de "los embriones del violeta", es decir una examinación del mundo simulado que produce el violeta y una contemplación sobre la naturaleza del violeta mismo, puede ser revelador en lo que concierne la perspicacia feminista de Gorodischer acerca de la estética del género (gender and genre).

El desarrollo de la ciencia y la maestría de su tecnología, debido al progreso social, han sido considerados una empresa masculina, modernizadora y primermundista. A su vez, la ciencia ficción dura, o "auténtica" (según algunos críticos literarios), lleva con ella un impulso imperialista que requiere una competencia tecnológica. ${ }^{1}$ Por esta

1 En su libro, A New Species: Gender and Science in Science Fiction, Robin Roberts, citando a Haraway, explica que la ciencia ficción dura: "[...] refers to fiction that focuses on technology where the fiction's hardware is scrupulously accurate: nothing in a piece of hard science fiction contradicts known scientific facts, at least the facts known at the time of the work's creation" [se refiere a la ficción que se enfoca en la tecnología donde el soporte físico de la ficción es meticulosamente acertada: nada en una obra de ciencia 
razón, las poblaciones que se encuentran alienadas o distanciadas de la tecnología y excluidas del control sobre su implementación tienden a identificarse más con los impactos sociales de la ciencia, en vez de hacerlo con los de la ciencia misma. No es de extrañar, entonces, que los críticos literarios hayan descubierto similitudes entre la ciencia ficción producida en contextos tercermundistas y la ciencia ficción escrita por mujeres y feministas. En general, aquellas "otras" ciencias ficciones están más íntimamente ligadas con lo que los teóricos categorizan como ciencia ficción "blanda", un subgénero con un énfasis en las implicaciones sociales de la ciencia y las ciencias sociales. En efecto, los escritores de ciencia ficción blanda exponen cómo los contextos sociales influyen en las interrogaciones e investigaciones científicas, las suposiciones que las apoyan, y el impacto social de los resultados consiguientes.

La definición de ciencia ficción según Darko Suvin podría resumirse con la frase "extrañamiento cognitivo", o mejor dicho, "the presence and interaction of estrangement and cognition" [la presencia e interacción del extrañamiento y del conocimiento] creado por " $[\ldots]$ an imaginative framework alternative to the author's empirical environment [novum]" [una infraestructura imaginativa y alternativa al ambiente empírico del autor] (7-8). El problema de clasificación de la ciencia ficción de América Latina tiene que ver más con el hecho de que "[...] la presencia de ese novum tecnológico o científico es escasa o inexistente en buena parte de la ciencia ficción latinoamericana y en particular en la argentina [...]" (Pron 64); en otras palabras, es difícil clarificar el aspecto científico o tecnológico de la narrativa, si entendemos la ciencia y la tecnología en términos de las ciencias duras y sus productos. A veces los críticos resuelven esta contradicción mediante la afirmación de que lo que escriben los latinoamericanos es, más bien, fantástico, o caracterizando a la ciencia ficción latinoamericana como metafísica, mítica o especulativa. ${ }^{2}$

ficción dura contradice hechos científicos, al menos aquellos hechos conocidos a la hora de la creación literaria] y acerca de las ciencias duras, clarifica que "The natural sciences are categorized as hard because they are ostensibly more objective and rigorous, while the social sciences are depicted as soft because, in this construction, they are supposedly more subjective and easier to master. These categories are coded through gender, as Sandra Harding points out in The Science Question in Feminism" [las ciencias naturales están categorizadas como duras porque son ostensiblemente más objetivas y rigurosas, mientras que las ciencias sociales están representadas como blandas porque, en aquella construcción, son supuestamente más subjetivas y fáciles de dominar. Estas últimas categorías están codificadas por el género (gender), como señala Sandra Harding en The Science Question in Feminism] (5). Angélica Gorodischer sugiere que "La ciencia ficción de Estados Unidos es una ciencia ficción imperialista o antiimperialista, pero siempre está el imperio ahí en el fondo, en el medio, siempre está la expansión imperialista" (Gorodischer en Ferman 47).

2 Según Pablo Capanna, “"En general, [los escritores latinoamericanos] cultivan una literatura fantástica no tradicional, que linda con la ciencia ficción, la atraviesa y sale libremente de su ámbito, con escasa presencia del elemento científico-tecnológico" (Capanna citado en Souto 19). Para Angela B. Dellepiane "Es a este nuevo tipo de ciencia ficción especulativa, a la que considero más fuertemente relacionada con la ciencia ficción argentina e hispanoamericana en general" donde "el escritor deja de lado la tecnología 
Entre otras cosas, estos críticos proponen que la literatura de ciencia ficción de la región se vincule más con los conflictos del hombre ante un mundo tecnológico. Angela B. Dellapiane articula esa idea elocuentemente: "Entiéndase que para la ciencia ficción la ciencia y la técnica son temas de los que se vale para concretar novelescamente el problema -no científico- del hombre versus esta civilización tecnolátrica en que parece que vive atrapado" (Dellapiane, "Narrativa argentina de ciencia ficción" 516). La crítica literaria Yolanda Molina Gavilán también observa el enfoque social de la ciencia ficción en Argentina, de manera tal que lleva "[...] an emphasis on issues related to humanity rather than technology" [un énfasis en asuntos de la humanidad en vez de la tecnología" (Gavilán 401). De la misma manera, en una entrevista reciente con Celia Esplugas, la escritora argentina de ciencia ficción y fantasía Angélica Gorodischer justifica el énfasis metafísico de la ciencia ficción en Argentina en el hecho de que sus habitantes están apartados de los últimos avances de tecnología:

Para nosotros, y digo nosotros porque en Argentina es mucha la gente que escribe ciencia-ficción, es imposible escribir eso que en los Estados Unidos se llama cienciaficción dura. En un país en el que no funcionan los teléfonos y donde tener un auto es un lujo, no podés andar escribiendo ciencia-ficción tecnológica ni explicando las naves que van a las estrellas ni hablando de imperialismos interestelares, por favor. Lo que sí hacemos es escribir algo que podría llamarse narrativa metafísica. (Esplugas 56)

Mientras que muchos críticos atribuyen la prevalencia de la forma blanda del género dentro del contexto latinoamericano, especialmente en Argentina, a “[...] la percepción de la ausencia de una tradición científica [...]" (66) o al acceso limitado a las tecnologías avanzadas, Patricio Pron sugiere que "Hay un elemento más en juego, a menudo no considerado por la crítica, y es la tradición 'anticientífica' de la literatura argentina, en la que la ciencia ficción producida en ese país se inscribe" (68). Pablo Capanna, un historiador eminente del género, reconoce el papel que juega la herencia literaria en el desarrollo de ciencia ficción "[...] son escritores que se han formado leyendo ciencia ficción y en cuyo mundo espiritual importan las convenciones y los mitos del género", y defiende la posibilidad de que en "Argentina, desindustrializada y estancada por la soberbia, la deshonestidad y la intolerancia de varias décadas, paradójicamente puede

como fin en sí mismo, subordinando consecuentemente la imaginación científica a un interés focal en las emociones y actitudes humanas personales así como también en problemas sociales" (Dellepiane, "Narrativa argentina de ciencia ficción" 515). Patricio Pron advierte que "no debería pues limitarse a la adopción de definiciones procedentes del ámbito anglosajón para leer la ciencia ficción producida en América Latina -como se hace habitualmente-, sino que debería más bien atender a la especificidad de las tradiciones nacionales en las que esa ciencia ficción latinoamericana se inscribe, la que escribieron Oesterheld y todos los otros" (72). 
llegar a encontrar una forma de expresión válida en esta literatura" (Capanna en Souto 19). Tal vez, resultaría ventajoso expandir el concepto del novum científico o tecnológico para incluir más creatividad e innovación en cuestiones de género (gender and genre). ${ }^{3}$

En Feminist Fabulation: Space/Postmodern Fiction, Marleen S. Barr plantea la noción de que la ciencia ficción feminista no es considerada como la ciencia ficción tout court cuando arguye que "Difference, women's impulse to power-not science-is at the heart of feminist SF" (3) y alude a la metaficción como su estética principal (8). A mi modo de ver, las motivaciones de la ciencia ficción feminista no pueden estar meramente reducidas a explorar los impactos de la ciencia y la tecnología, sino más bien a explorar el papel que juega el proceso científico en producir la sabiduría sobre la diferencia, que tiende a ser la base de intervenciones y reconfiguraciones feministas. Además, una lectura feminista de la supuesta distinción entre las ciencias duras y las ciencias sociales revela ciertas tensiones: 1) la separación artificial entre la ciencia y sus impactos sociales; 2) la falta de conocimiento acerca de la producción social de los hechos científicos; y 3) la complejidad de la interrelación y la interacción entre los seres humanos y la tecnología misma. Robin Roberts, en su estudio A New Species: Gender and Science in Science Fiction, provee un ejemplo de las dificultades que se producen al mantener ese binario:

The distinction between hard and soft science, however, turns out to be difficult to maintain, particularly when hard science is practiced in relation to reproduction. For example, although new technologies involving human reproduction are clearly hard science, that is, they rely on machinery and biology, these technologies have been characterized as soft, in part because of their immediate implications for social organization and also, perhaps, because of their association with women. $(5-6)^{4}$

3 La misma Gorodischer reflexiona sobre sus actos creativos acerca de la ciencia: "Entonces empecé a escribir ciencia ficción, una ciencia ficción muy especial, no tecnología y nada conectada con la ciencia (porque yo de ciencia no sé un pito a la vela). Pero también hay maneras de inventar la ciencia, hay maneras de inventar las matemáticas y todo eso yo lo he puesto en mis libros" (Gorodischer en Fermán 49). Patricio Pron destaca el hecho de que "El concepto de 'innovación científica' es relativo: para algunas sociedades lo es la posibilidad de enviar científicos a Marte, para otras lo es el acceso a vacunas" (73). Según Yolanda Molina Gavilán, "The violet circles-a central focus of this story-may be regarded, indeed, as one of the fantastic or magical elements of Gorodischer's fiction that Souto and Mosier mention. On the other hand, some of the characters of the story attempt to decipher the origin of the circles in rational, logical terms" [Las manchas violeta -un enfoque central del cuento- pueden ser consideradas, de hecho, como uno de los elementos fantásticos o mágicos de la ficción de Gorodischer que mencionan Souto y Mosier. Por otro lado, algunos de los personajes del cuento intentan descifrar el origen de las manchas en términos racionales y lógicos] (404-405). Pablo Capanna también sustenta el carácter enigmático de su narrativa: "Aun la obra de Angélica Gorodischer, quien manifiesta su afiliación al género, difícilmente podría encasillarse en sus normas más ortodoxas" (Capanna citado en Souto 19).

4 [La distinción entre la ciencia dura y blanda, sin embargo, se torna difícil de mantener, en particular cuando la ciencia dura está relacionada con la reproducción. Por ejemplo, aunque las nuevas tecnologías 
De hecho, propongo que el tema principal de "Los embriones del violeta" es la reproducción, y por su parte el novum tecnológico del violeta nos hace contemplar cómo nuestras concepciones acerca de la reproducción ayudan a construir las diferencias entre los sexos, y cómo el significado de la humanidad misma está imbuido en la reproducción biológica en nuestro mundo actual.

Podría dejar al lector perplejo el saber que Gorodischer, quien sostiene que "En la ciencia-ficción es la humanidad toda la que está involucrada" "Narrativa fantástica" 49), más aún cree un mundo sin mujeres en el que jerarquías de poder relacionadas con el género todavía existen. No sólo se refiere a los tropos tradicionales de la ciencia ficción dura en su obra, sino que también ella añade técnicas metanarrativas e ideas metafísicas, ambas típicas de la ciencia ficción feminista y latinoamericana. "Los embriones del violeta" relata una misión exploratoria de la tripulación de la nave Luz Dormida Tres que redescubre el planeta de Salari II, que se pensaba desierto. Para sorpresa de los personajes (y del lector), el extrañamiento cognitivo ("cognitive estrangement") en esta obra de ciencia ficción no es el de reconocer la otredad, sino la semejanza. Es precisamente la similitud de Salari II con la tierra lo que confunde a los exploradores del espacio sideral. ${ }^{5}$ El hecho de que la similitud (en lugar de la diferencia) vuelve a ser la fuerza alienígena y ajena de la narrativa refleja un mundo en que la mujer no existe como "la otra" opuesta al hombre. La población del planeta, constituida solamente de hombres, representa el deseo de mantener una jerarquía entre los géneros por el deseo de producir la diferencia de la semejanza. Aún los hombres producidos por el violeta, quienes hacen papeles de mujeres, representan unas tentativas para llenar el vacío de la otredad. Además, el estilo de la narrativa, que se caracteriza por la escasez de transiciones e indicaciones sobre cambios de tiempo y lugar, da la impresión de una simultaneidad. Además vuelve más confuso determinar qué miembros pertenecen a cada tripulación, cuáles son los nombres originales de los habitantes de Salari II y cómo se correlacionan con los nombres inventados, y, por fin, cuáles de ellos vienen del violeta y cuáles tienen origen en la Tierra.

La ausencia de mujeres no solamente se vuelve problemática para la clasificación genérica del texto, sino que también para los críticos que intentan clasificar la infraestructura genérica del cuento y el papel enigmático de las manchas violeta. ${ }^{6}$ Inspirado en el planeta

para la reproducción humana son ciencia dura, es decir, dependen de la maquinaría y la biología, estas tecnologías han sido caracterizadas como blandas, en parte por causa de las implicaciones inmediatas para la organización social y también, tal vez, porque están asociadas con las mujeres] (mi traducción).

5 Beatriz Urraca tiene un ensayo brillante en que plantea "Los embriones del violeta" como una narrativa alternativa de la conquista del nuevo mundo, y su forma de extrañamiento como parte integral de su estética. Urraca, Beatriz. "Angelica Gorodischer's Voyages of Discovery: Sexuality and Historical Allegory in Science-Fiction's Cross-Cultural Encounters." Latin American Literary Review 23.45 (1995): 85-102. JSTOR. 20 sep. 2010.

6 Ver la segunda mitad de este ensayo para una elaboración del asunto. 
de Solaris de Stanislaw Lem, el Salari II diseñado por Gorodischer se parece al planeta original, donde el océano emerge como una fuerza femenina protegiéndose contra la colonización al fabricar manifestaciones físicas de la culpa de sus invasores. ${ }^{7}$ En el Salari II, sin embargo, los miembros de la tripulación varada del Niní Paume Uno se creen dioses por el poder creativo que derivan de las manchas violeta dispersas en la superficie del Salari II. Para generar del violeta un objeto de deseo existen dos condiciones: 1) el individuo debe poder imaginarse la encarnación del objeto que desea poseer (de tal manera que tiene que deshumanizarse) y 2) nada creado por el violeta puede salir del planeta Salari II. ${ }^{8}$ Aunque la tripulación varada del Niní Paume Uno parece tener el control de sus creaciones, el verdadero poder creativo pertenece al violeta. Ciertamente, las manchas violeta sólo generan objetos que llenan el verdadero deseo del individuo, lo cual implica que el poder creativo depende del discernimiento del violeta acerca de la autenticidad del deseo. Un "embrión" del violeta, propongo, no es simplemente un simulacro producido a partir del deseo de uno de los miembros de la tripulación. ${ }^{9}$ Las manifestaciones del deseo también demuestran la naturaleza de su carácter moral, a su vez reduciéndole a una esencia singular. Cada miembro de la tripulación varada, de hecho, encarna un complejo psicológico, y los mundos que crean exponen sus luchas internas y privadas al resto del mundo: la dialéctica amo-esclavo, el retorno a la matriz, el sadomasoquismo, el olvido caprichoso, y la búsqueda del conocimiento.

$\mathrm{Al}$ escribir lo que a primera vista parece ser ciencia ficción dura con un reparto enteramente masculino, incluyendo a los personajes que no consiguen percibir el poder y la ingenuidad de las manchas violeta, Gorodischer impide cualquier clasificación fácil de su cuento como ciencia ficción dura o blanda. Por ejemplo, Leo Sessler, un intelectual y "hombre de ciencia", que tiene el papel de cronista en la expedición espacial del Salari II, todavía no entiende o no puede captar la complejidad del violeta, ejemplificado en su pregunta a Vantedour: "qué piensa usted de este fenómeno de conseguir cosas de la nada [131]". El hecho de que Sessler no pueda nombrar, clasificar, o entender el origen o la naturaleza de las manchas violeta, revela una falla de principios científicos para proveer una explicación racional que típicamente sustenta a las narrativas de la ciencia ficción dura. De otra manera, se puede reconocer la presencia de lo que Patricia Melzer califica como "alien constructions" [construcciones ajenas/alienígenas] en la ciencia

\footnotetext{
Angela B. Dellepiane confirma que "Se le encuentran reminiscencias temáticas del Solaris de Stanislaw Lem, y una complejidad estilística sólo comparable a la norteamericana Ursula Le Guin y a la de la francesa Natalie Henneberg, ambas muy leídas y admiradas por AG" (Dellepiane, "Boca de dama" 21).

8 Olga Juzyn-Amestoy tiene un análisis importante acerca de esta primera condición, en el que sugiere que "se trata de una estrategia afín a la empatía" (92).

9 Muchos teóricos comentan acerca de la idea de que lo que produce el violeta es un simulacro. De hecho, la presencia de los dos soles le hace al lector acordarse de la trama de La invención de Morel por el argentino Adolfo Bioy Casares, en que el protagonista aprende que el amor de su vida es una mera proyección filmada del pasado superpuesta sobre la tierra de la isla que habita.
} 
ficción, o mejor dicho, "metaphors and concepts specific to the genre" [metáforas y conceptos relacionados al género] (Melzer 11). Para ella, la ciencia ficción feminista depende de " $[t]$ hese alien constructions, embedded within a narrative context that enables identifications, can provide us with empowering metaphors that allow critical evaluations of the theories we rely on to explain our social realites" [aquellas construcciones alienígenas, enclavadas dentro de un contexto narrativo que facilita identificaciones puede proveer metáforas inspiradoras que dejan evaluar críticamente las teorías con las cuales contamos para explicar nuestras realidades sociales] (Melzer 11). Es precisamente en estas construcciones alienígenas que deseo concentrar mi análisis, es decir, en la esencia de los embriones producidos por el violeta, en la función y la construcción textual del violeta por Gorodischer, y en cómo ambas reproducciones nos ayudarían a abordar una teoría de la ciencia ficción más inclusiva.

“[P]ORQUE TODO ESTO ERA UNA BELLEZA MATERNAL NO GARANTIZABA UNA ACOGIDA AMISTOSA"10

En la literatura secundaria sobre "Los embriones del violeta" y la colección de cuentos de la que forma parte, Bajo las jubeas en flor se destacan algunos críticos literarios que pueden propiciar una interpretación más abarcadora de la teoría del género (gender and genre) en el texto. En "Angélica Gorodischer y Jorge Luis Borges: La ciencia ficción como parodia del canon", Luis Cano propone que la narrativa de Gorodischer, dentro de la tradición Borgesiana, revela "un intento de parodiar una tradición literaria predominantemente masculina, desde el interior de un género eminentemente masculino" (458). ${ }^{11}$ A su vez, Beatriz Urraca, en "Angelica Gorodischer's Voyages of Discovery: Sexuality and Historical Allegory in Science-Fiction's Cross-Cultural Encounters", sostiene que Gorodischer rompe con las convenciones literarias en la tradición de la ciencia ficción feminista que usualmente reemplaza ciencia ficción dura (en la que lo masculino ocupa un lugar central) con una ciencia ficción blanda (en la que configura lo femenino a su meollo). Lo que ambos críticos añaden a la conversación acerca de la estética de Gorodischer es una contemplación por parte de la misma sobre el género (gender) del género (genre); es decir que la infraestructura y el contenido del texto

${ }^{10}$ Gorodischer, "Los embriones del violeta" 120.

11 Acerca de la carencia de mujeres en la colección de cuentos de Gorodischer, Luis Cano elabora que "La casi total ausencia de mujeres en las narraciones del volumen y su representación estereotípica en los relatos interpolados en 'Jubeas', efectúa una parodia de la tradición de ciencia ficción escrita por hombres y orientada a un consumidor masculino" (459). Beatriz Urraca también observa que "Gorodischer defines herself as a feminist, and though some of her stories may lack female characters, it is also possible to read in them a deep interest for women's concerns" [Gorodischer se declara feminista, y aunque algunos de sus cuentos carecen de personajes femeninos, es también imposible de interpretar un interés profundo en las preocupaciones de las mujeres en sus textos literarios] (85). Lefanu tiene un argumento similar sobre la escritora eminente de ciencia ficción norteamericana, Ursula K. Le Guin. 
mismo reflexionan metatextualmente sobre las normas de escritura y su afiliación al género, en ambos sentidos de la palabra. ${ }^{12}$ Parece que las normas lingüísticas y las formas narrativas típicas de la ciencia ficción dura y la ciencia ficción feminista sean deficientes para expresar su visión del mundo.

En el ensayo "Las mujeres y las palabras", Gorodischer propone una alternativa a la división simplificada entre la escritura masculina y la escritura femenina, una división basada en un esencialismo problemático. Para Gorodischer, las mujeres manejan un "lenguaje del exilio" porque ocupan posiciones marginales en la sociedad patriarcal, pero también ve la importancia de tener como objetivo un "lenguaje integrado":

Lo que hay que incorporar es el lenguaje del exilio, que no responde a los mitos de "naturaleza femenina" sino a la realidad impuesta de una marginalidad sin sentido, para que alguna vez el lenguaje sea uno y completo.

Lo ideal y lo necesario sería no la combinación o la mezcla, sino la interacción de ambos lenguajes, el masculino de poder, de acción personalizada, y el femenino mítico, de exilio y marginalidad, y usar esas palabras así, hasta que la distinción se perdiera. (47)

Resulta importante la noción de "lenguaje del exilio" porque sugiere que en "Los embriones del violeta" el extrañamiento cognitivo ocurre a través de una forma de auto-exilio de la Tierra por parte de los habitantes del Salari II y un lenguaje heredado que no es suficiente para captar la diferencia del contexto en el Salari II. ${ }^{13}$ Es decir, que el Salari II ofusca la esencia verdadera de las construcciones del mismo y del otro, y revela la autodestructividad de tales maneras binarias de pensar. Como resultado, el

12 Con mucha perspicacia, Urraca aclara que “Gorodischer's work breaks several stereotypes of feminist science-fiction. Here we find no all-female utopias, no 'women wielding magical powers and defeating male-dominated technology,' no powerful female characters or sympathetic female aliens, [...] no role models for women in society [...]-for her stories completely lack women. She dares to imagine a world like ours, in which a few crucial changes make it possible for us to see what encumbers us" [La obra de Gorodischer rompe con muchos estereotipos de la ciencia ficción feminista. Aquí no encontramos utopías femeninas, 'mujeres ejerciendo poderes mágicos y conquistando una tecnología creada por hombres', personajes femeninos poderosos o simpáticos extraterrestres femeninos, modelos a imitar para las mujeres en la sociedad - porque sus cuentos carecen completamente de mujeres. Ella se atreve a imaginar un mundo parecido al nuestro, en la que pocos cambios cruciales hacen posible para nosotros ver lo que nos abruma] (99). Cano también comenta sobre esta inversión de ciencia feminista en la obra de Gorodischer (459).

13 Para Beatriz Urraca, "As the discoverer in charge of the linguistic aspect of the operation, Sessler's self-imposed task is to name and rationalize the new worlds as an act of possession through language. Yet Salari II escapes words as his language proves inadequate, and he is impotent to explain, define, and control the new reality" [Como descubridor a cargo del aspecto lingüístico de la operación, la tarea auto-impuesta de Sessler es nombrar y racionalizar los nuevos mundos como un acto de posesión por el lenguaje. Pero el Salari II elude las palabras en tanto su lenguaje se revela inadecuado, y él es impotente para explicar, definir, y controlar la nueva realidad] (92). 
lenguaje del exilio en "Los embriones del violeta" expresa "la realidad impuesta de una marginalidad sin sentido" al poner a los hombres en posición de exilio e incluirlos en espacios femeninos, al exteriorizar el espacio interno y desafiar el modelo de penetración para llegar al conocimiento, y en consecuencia, subvertir la dicotomía entre la superficie y la profundidad. ${ }^{14} \mathrm{Al}$ respecto, Gorodischer parece proponer que el lenguaje femenino como lenguaje del exilio es, en realidad, un lenguaje de ciencia ficción.

En "Los embriones del violeta" Gorodischer llama la atención al lenguaje femenino del exilio a través de la metáfora del violeta. Según Gorodischer las manchas violeta, "Tienen bordes imprecisos y parecen fluctuar, moverse, están vivas tal vez, y tal vez son mortíferas" (125-126). Si interpretamos la metáfora de las manchas violeta como una forma de teorizar el género (gender), la idea de que las categorías del género [gender] "tienen bordes imprecisos" sugiere la posibilidad de unas definiciones fluidas, flexibles, y móviles. Por un lado, tienen vidas propias (o mejor dicho que las categorías del género cambian, dependiendo del ambiente social y las circunstancias) y por otro lado, pueden tener consecuencias mortíferas (es decir que muchas veces se usa la violencia para mantener la distinción entre ellas).

El violeta, al crear reflejos de origen ambiguo, también evita ser reducido a sus orígenes y nos hace pensar en cómo nuestros modos de ver revelan algo esencial sobre nosotros mismos. La siguiente cita ilustra este concepto: “[...] el violeta seguía allí extendiéndose hacia abajo pero no como una cualidad de la tierra sino como un reflejo. Solamente que si usted, parado allí, busca la fuente de ese reflejo, hacia arriba y hacia los costados, no encuentra nada. Permanecen, un poco fluctuantes siempre, también de noche, o sobre la nieve cuando nieva. No sabemos que son ni qué tienen" (132). Esta última declaración resalta que no se puede entender el violeta ontológicamente ni por lo (tal vez, el poder) que posee. De esa manera Gorodischer descalifica unas maneras comunes para conocer y categorizar a la otredad, los cuales se usan para también diferenciar entre los géneros. La metáfora del violeta también resuena con la descripción de la mujer dentro del sistema patriarcal, según Gorodischer, "Como eco, como reflejo, como objeto del hombre, [quien] no habla, [quien] no es sujeto" ("Las mujeres y las palabras" 46). Al comparar los dos textos de Gorodischer, uno de ciencia ficción y otro de ensayo, las manchas del violeta, incorpóreas y reproductoras, hacen referencia al patrón en que la mujer, definida por su capacidad de reproducir la vida humana, vuelve a

14 Para elaborar este concepto, Gorodischer dice: "Ese lenguaje integrado, masculino y femenino, debe conservar la afectividad al lado de la lógica y la acción, terminar con la dicotomía cuerpo-espíritu, incluir el cuerpo omnipresente de la mujer que cierra el camino a la sublimación cuando se convierte en objeto deseado y en objeto deseado por la que desea al que desea, incluir la cabeza del hombre que excluye al cuerpo y trabaja por su cuenta, abordar los temas tabú y las palabras prohibidas, dejar hablar al interior y no al exterior asiento del espejo, solamente. Hay que emerger de esa exterioridad, de esa apariencia ungida de sagrada, y sobre todo, hay que llegar a la inclusión del hombre en los espacios femeninos" ("Las mujeres y las palabras" 47-48) 
ser reducida a su cuerpo. De tal manera, produce un sentido de extrañamiento cognitivo cuando abstrae la idea del cuerpo en la metáfora y divulga cómo funciona el género sin la necesidad del cuerpo.

Olga Juzyn-Amestoy aclara el significado de la metáfora del cuerpo femenino y como funciona: "Como una viva metáfora el cuerpo femenino (en su función sexual y maternal) nos trae de vuelta de ese lugar donde le buscamos el sentido a las palabras; nos hace ver que es en la gestación misma que nos afirmamos y abrimos paso en la historia. Nuestro cuerpo esconde el secreto del lenguaje: está habitado por lo desconocido, una ausencia" (93). Entonces, en qué espacio "le buscamos el sentido a las palabras" si la narrativa de Gorodischer carece del cuerpo de la mujer (pero no de cuerpos feminizados)? ${ }^{15}$ Si no lo podemos encontrar adentro del cuerpo femenino, tal vez lo podemos encontrar en los espacios exteriores, en los espacios siderales, o en el mundo que nos rodea. Por su parte, Gorodischer acepta como punto de partida un mundo estable en el cual "los cambios se producen en la superficie" (49) o sea que "[e]sta realidad que me rodea es algo que voy inventando, que me pertenece sólo en parte, que cambiaría si yo no estuviera, que es un poco interna y un poco externa, que yo, y conmigo todos, he y hemos cubierto de palabras" ("Narrativa fantástica y narrativa de ciencia-ficción" 48). Es decir que nuestro mundo, en el que las distinciones entre adentro y afuera son borrosas, está inventado individualmente y colectivamente a través del lenguaje.

En otro ejemplo, el violeta no inspira mucha emoción: “Cuando miré hacia abajo, la carpa y el infinito mundo pardo que empezaba a iluminarse con los dos soles, y las manchas violeta que parecían agua, o aguas vivas, pensé que, con todo, era una lástima. No tenía miedo, no me daba miedo eso de morir, porque no pensaba en la muerte" (127). Aquí, el acto de contemplar al violeta por parte de Vantedour, invoca el sentimiento de lástima, pero lástima ¿de qué? Al principio parece que la lástima podría ser que estuvieran destinados a morir en el Salari II. Pero, al mirar nostálgicamente a este momento, Vantedour parece lamentar que los secretos del violeta quedan inviolados, ¿que no puede descifrar el significado del violeta, o que depende del poder reproductivo del violeta? Aunque el violeta niegue la posibilidad de engendrar a las mujeres, y por extensión, a los hijos, Vantedour todavía no tiene miedo de la inevitabilidad de su propia muerte. Quizás podemos interpretar este sentido falso de seguridad como una forma de

15 Urraca entiende como reproducen en el Salari II las estructuras de dominación parecidas a las del patriarcado cuándo explica que: "As a narrative strategy, the absence of women in her stories draws attention to a world dominated by sexism: Gorodischer forces us to imagine a world in which the female as object of oppression has disappeared, and the resulting configurations of space and culture produce different patterns of dominance and adaptation" [Como una estrategia narrativa, la ausencia de las mujeres en sus cuentos llama la atención a un mundo dominado por el sexismo: Gorodischer nos esfuerza a imaginar a un mundo en el que la mujer como objeto de opresión ha desaparecida, y los consiguientes configuraciones del espacio y cultura produce patrones diferentes de dominancia y adaptación] (87). 
distracción producida por el violeta para los habitantes del Salari II, quienes no pueden percibir a la verdadera amenaza entre sus simulacros deseados. Esta interpretación está apoyada por la observación de Sessler, a quien cito en el epígrafe de la segunda mitad de este ensayo: "[p]orque todo esto era una belleza maternal no garantizaba una acogida amistosa" ("Los embriones del violeta" 120). La supuesta belleza maternal de la tierra y el pretendido control sobre la tecnología del violeta parecen ser unas tretas para engañar a los descubridores y también unas estrategias anticolonizadoras. ${ }^{16}$

Finalmente, Vantedour compara el violeta con la certidumbre de la muerte y, quizás, de su propia locura: "Como la muerte -dijo el Señor de Vantedour-, esto era algo que había que aceptar. Y aun cuando estuviéramos locos, si podíamos fumarnos nuestra locura, afeitarnos con nuestra locura, llenarnos el estómago con nuestra locura, era no sólo conveniente sino necesario aceptarlo" (127-128). Lo que sugiere la susodicha cita es que los miembros de la tripulación del Niní Paume Uno tuvieron que aceptar como realidad a los simulacros creados por el violeta para sobrevivir, a pesar de que eso signifique su demencia. Es significativo que "los cambios se producen en la superficie" del planeta Salari II y que este mundo está inventado por sus propios habitantes, como en "La narrativa fantástica y la narrativa ciencia-ficción" arriba mencionado de Gorodischer. Además, cada uno de los habitantes del Salari II tiene que mantenerse en el estado de exilio (o, según Gorodischer, el estado de la mujer) para conservar el mundo simulado del violeta. Urraca también advierte al lector: "This paradise must not be confused with a utopia: there is no social organization, no possibility of species survival, and the reader fails to empathize with a concept of male happiness the story puts forth as negative and unproductive" [Este paraíso no se puede confundir con una utopía: no hay organización social, no existe posibilidad de sobrevivencia de la especie, y el lector falla en empatizar con un concepto de la felicidad masculina que el cuento propone como negativo y no productivo] (95). De hecho, la sustitución de la reproducción biológica con la simulación garantiza que la genealogía humana en el Salari II terminará con la muerte de la tripulación varada del Niní Paume Uno.

Si tomamos en cuenta esa idea de que el mundo del Salari II es femenino en su naturaleza y que el violeta es una fuerza feminizadora, parece interesante que el violeta imparta la posibilidad de crear mundos deseados pero niega el poder de materializar la mujer. Según Urraca, "[Gorodischer] alienates her male narrators and their masculine missions through a feminization of the new, imaginary worlds and their seemingly male inhabitants" [[Gorodischer] aliena a sus narradores y sus misiones masculinas a través de una feminización de los nuevos mundos imaginarios y sus habitantes

\footnotetext{
${ }^{16}$ Aunque Juzyn-Amestoy comenta acerca del género lingüístico del violeta, a mi modo de ver no refleja necesariamente que es una fuerza masculina: "Debidamente de género masculino, el don de reproducción en el cuento de Gorodischer -el violeta- es creador de simulacros" (88).
} 
supuestamente masculinos] (86). Es decir que la feminización sólo funciona al nivel abstracto por convertir a los descubridores del planeta en otros; en efecto, los deseos de los descubridores ayudan a construir sus esencias, pero nunca están reducidos a sus cuerpos y siguen teniendo dependencia del violeta para hacer realidad los sueños. A nivel social, los seres creados por el violeta toman papeles típicamente femeninos para satisfacer a los apetitos de los miembros de la tripulación del Niní Paume Uno. No queda claro, sin embargo, si son seres autónomos o autómatas; en cualquier caso, tienen posiciones subordinadas a los deseos de sus creadores.

Como "an alien construction" [una construcción ajena/alienígena], el violeta es una de estas "empowering metaphors that allow critical evaluations of the theories we rely on to explain our social realites" [metáforas inspiradoras que dejan evaluar críticamente las teorías con las cuales contamos para explicar nuestras realidades sociales] (Melzer 11). Es decir que el violeta y lo que produce una estética feminista que desafía la rigidez de las categorías del género (gender y genre). Tal vez podríamos interpretar a las manchas violeta (y el violeta mismo) -con sus bordes imprecisos y fluctuantes, las contemplaciones existenciales que inspiran, y los reflejos de origen ambiguo que crean- como metáfora no sólo del género [gender] sino del género [genre] de ciencia ficción. La ciencia en "Los embriones del violeta", en su encarnación masculinista e imperialista, falla en descubrir el origen de las manchas violeta y Gorodischer, en su lugar, no dirige la atención del lector hacia su esencia, sino que lo que las manchas reflejan, es decir, a los habitantes del Salari II y a los lectores mismos. Al tomar en cuenta la idea de Melzer de que la ciencia ficción provee un campo exploratorio para la teoría feminista, ${ }^{17}$ Gorodischer nos hace reflexionar sobre los mundos de ciencia ficción que inventamos - cómo podemos entender algo sobre el mundo que nos rodea al verlo desde un punto de vista alienígena, y a la vez o al mismo tiempo que los mundos que seguimos inventando reflejan algo interesante sobre nuestros propios deseos-. Al combinar y confundir los espacios interiores y exteriores, la distinción entre la ciencia ficción dura y la ciencia ficción blanda, las estéticas típicamente feministas y masculinas, Gorodischer le hace al lector recordar que "[...] la ciencia-ficción tiene una orientación cósmica, que falta o no es necesaria en otras narrativas fantásticas. En la ciencia-ficción es la humanidad toda la que está involucrada" ("Narrativa" 49).

${ }^{17}$ Según Melzer, "Science fiction's fantastic aliens and distant planets can thus become the imaginative testing grounds for feminist critical thought. These texts create a link between cultural imagination and political positions: they function as 'case studies' of how feminist theories 'work" [Los extraterrestes fantásticos de ciencia ficción y los planetas lejanos pueden transformarse en un campo imaginativo de pruebas para el pensamiento crítico sobre el feminismo. Estos textos crean un vínculo entre la imaginación cultural y las posiciones políticas: funcionan como 'casos prácticos' de cómo 'funcionan' las teorías feministas" (11). 


\section{BiBLIOGRAFÍA}

Barr, Marleen S. Feminist Fabulation: Space/Postmodern Fiction. Iowa City: U of Iowa P, 1992.

Cano, Luis C. "Angélica Gorodischer y Jorge Luis Borges: La ciencia ficción como parodia del canon”. Hispania LXXXVII/3 (2004): 453-63.

Dellepiane, Angela B. "Contar $=$ mester de fantasia o la narrativa de Angélica Gorodischer”. Revista Iberoamericana LI/132-133 (1985): 627-40.

"La narrativa de Angélica Gorodischer". Boca de dama: la narrativa de Angélica Gorodischer. Miriam Echeverría Balboa y Ester Gimbernat Gonzalez, eds. Buenos Aires: Feminaria Editora, 1995. 17-40.

Gorodischer, Angélica. "Los embriones del violeta". Bajo las jubeas en flor. Buenos Aires: Ediciones de la Flor, 1973. 113-152.

"Entrevista con Celia Esplugas". Hispamérica XXIII/67 (1994): 55-59.

“Las mujeres y las palabras". Hispamérica XIII/39 (1984): 45-48.

"Narrativa fantástica y narrativa de ciencia-ficción". Plural: Revista Cultural de Excelsior 188 (1987): 48-50.

Juzyn-Amestoy, Olga. "La narrativa fantástica de Angélica Gorodischer: la mirada 'femenina' y los límites del deseo". Letras Femeninas (1994): 87-96.

Lefanu, Sarah. Feminism and Science Fiction. Bloomington: Indiana UP, 1988.

Melzer, Patricia. Alien Constructions: Science Fiction and Feminist Thought. Austin: U of Texas P, 2006.

Molina Gavilán, Yolanda. "Alternative Realities from Argentina: Angélica Gorodischer's 'Los embriones del violeta." Science Fiction Studies XXVI/3 (1999): 401-11.

Mosier, M. Patricia. "Women in Power in Gorodischer's Kala Imperial." In Spectrum of the Fantastic. Donald Palumbo, ed. Westport, CT: Greenwood, 1988. 153-61.

Pron, Patricio. "¿Es posible una ciencia ficción sin ciencia? La literatura argentina fantástica y de ciencia ficción ante el abismo tecnológico". Revista de Occidente 365 (2011): 61-75.

Roberts, Robin. A New Species: Gender and Science in Science Fiction. Urbana: U of Illinois P, 1993.

Souto, Marcial. Introducción. La ciencia ficción en la Argentina: antología critica. Buenos Aires: EUDEBA, 1985.

Suvin, Darko. Metamorphoses of Science Fiction: On the Poetics and History of Literary Genre. New Haven: Yale UP, 1979.

Urraca, Beatriz. "Angelica Gorodischer's Voyages of Discovery: Sexuality and Historical Allegory in Science-Fiction's Cross-Cultural Encounters." Latin American Literary Review XXIII/45 (1995): 85-102. 
Uzín, María Magdalena. "Murmullos femeninos en la ciencia-ficción argentina: problemas de Gender y Genre". Revista Iberoamericana LXXVIII/238-239 (2012): 247-58. 